\title{
Pulsating stars and the Virtual Observatory
}

\author{
Juan Carlos Suárez ${ }^{1,2, \star}$ \\ ${ }^{1}$ Universidad de Granada (UGR), Dept. Theoretical Physics and Cosmology, 18071, Granada, Spain \\ ${ }^{2}$ Instituto de Astrofísica de Andalucía (CSIC), Glorieta de la Astronomía s/n, 18008, Granada, Spain
}

\begin{abstract}
Virtual Observatory is one of the most used internet-based protocols in astronomy. It has become somewhat natural to find, manage, compare, visualize and download observations from very different archives of astronomical observations with no effort. The VO technology beyond that is now being a reality for asteroseismology, not only for observations but also for theoretical models. Here I give a brief description of the most important VO tools related with asteroseismology, as well as a rough outline of the current development in this field.
\end{abstract}

\section{Why Virtual Observatory?}

In the last century astronomy has witnessed an exponential growth in many aspects: number of researchers, ground and space observatories, observation networks, surveys, etc. This has led to a significant advance in many fields of astrophysics. As a consequence an unprecedented improvement of the astronomical instrumentation has occurred. Such an improvement often refers to the quality and also the quantity of the raw data generated. Such an exponential growth (Fig. 1) will soon reach the limits of the data rates exchange and storage capacity of today's technology. The epitome of such saturation scenario is the Square Kilometer Array (SKA) project ${ }^{1}$.

Additionally, the increasing number of laboratories and instruments has also led to deal with different types of data structures and data files, each of them requiring a specific interface to be acquired with different computer languages and under different operating systems. Consequently, scientists spend a non-negligible amount of time preparing interfaces and adapting their own codes to be compliant with all different data sources. The Virtual Observatory (VO) was born to solve those problems, seeking to allow the exploration of astronomical data in a single transparent way, providing online powerful analysis and visualisation tools. One of the keys for success of this project is the philosophy of standardisation. This applies to the data, metadata, and the communication protocols among archives and VO tools. As well, the use of a registry. As stated in the International Virtual Observatory Alliance ${ }^{2}$ (sic) "The long term vision is not one of a fixed specific software package, but rather one of a framework which enables data centers to provide competing and co-operating data services, and which enables software providers to offer a variety of compatible analysis and visualization tools and user interfaces. The first priority for the VO projects worldwide is to develop the standardized

\footnotetext{
^jcsuarez@ugr.es

${ }^{1}$ http: //skatelescope.org

${ }^{2}$ The international organism that debates and agrees the technical VO standards.
} 


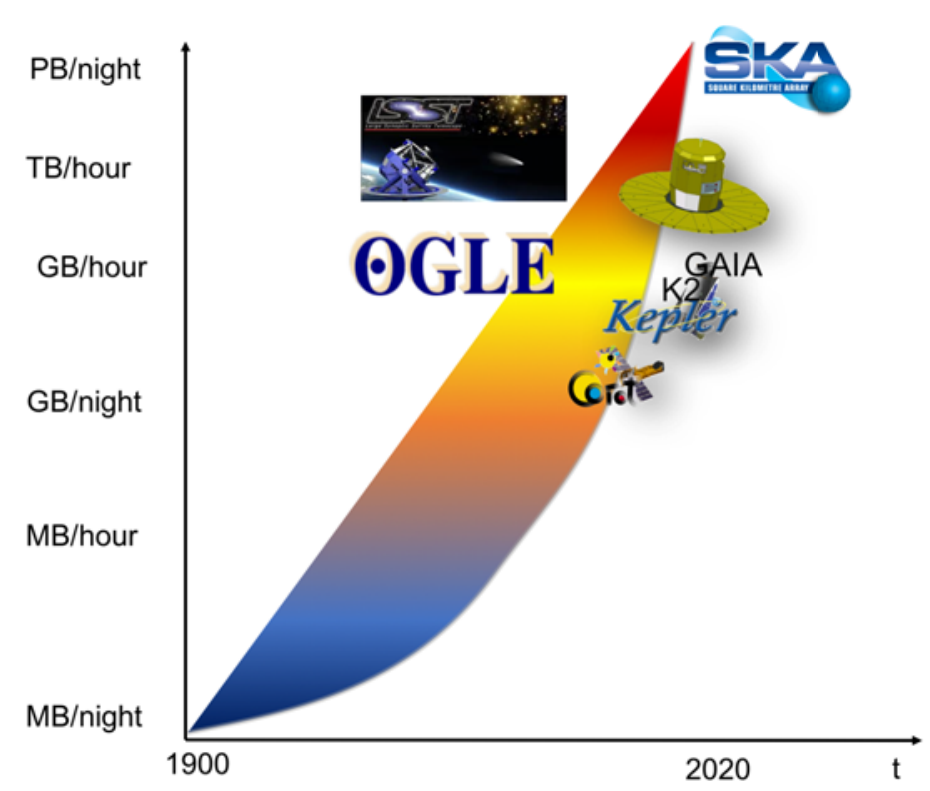

Figure 1. Evolution of the data rates produced by astronomical instrumentation. Notice that SKA in full operation will overwhelm the scale. The use of aperture array radio telescopes in the low and mid frequency ranges will imply data rates to many Petabits $\left(10^{15}\right)$ per second, which represents more than 10 times the current global internet traffic.

framework, which will allow such creative diversity". Today, VO projects are being developed worldwide, seeking full interoperability and standardisation among applications, archives and researchers, boosting the time spent by scientists for research ([1]).

Stellar pulsations have become a revolution in the field of stellar physics, and a major contributor to the field of exoplanetary science. This can be understood by the synergies (both instrumental and scientific) between asteroseismology and planet detection and characterization. Space missions like MOST ([2]), CoRoT ([3]), and Kepler ([4]) have provided thousands of light curves (time series of stellar luminosities). Those missions, which were designed mainly to detect planetary systems, use asteroseismology as the main technique to disentangle between signal coming from stellar pulsations and from the planetary transits (or even stellar activity). Space missions require complementary ground-based observations to fully accomplish their scientific objectives. This implies large international networks able to get the extremely competitive observation time in the observatories around the world. All this activity has increased exponentially the amount of data generated in the field of stellar pulsations, scenario that will get worse in the near future with the next-generation missions, namely CHEOPS ([5]), TESS ([6]), and PLATO ([7]).

Likewise, interoperability and data access has also changed dramatically for this field thanks to the VO. This has led to an entry into the era of big data, in which data discovery from automated procedures, statistical inferences and artificial intelligence (AI) have become common practice in the field ([8]). This allows to connect the information of many astrophysical surveys whose main scientific case are not necessarily related to stellar pulsations. We find many examples of this at the $22^{\text {nd }}$ Stellar Pulsation Conference (Fig. 2) like the Gaia survey in full operation $([9,10])$, which is provid- 


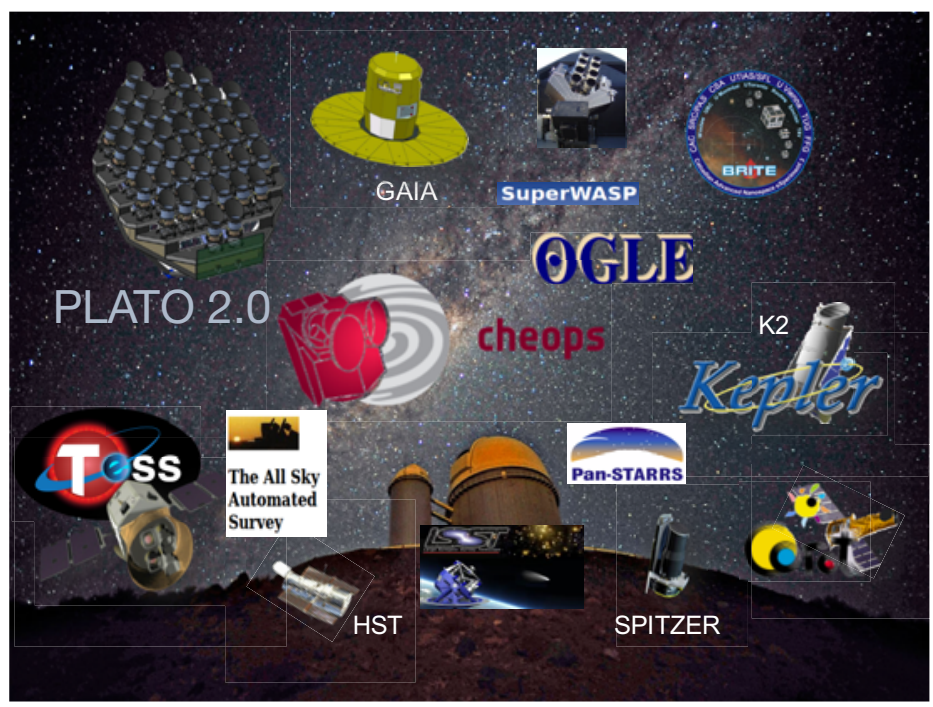

Figure 2. The instruments here schematically shown represent a sample of the current panoply of missions (flying and/or in preparation and/or in legacy mode) related to stellar physics and asteroseismology. Likewise groundbased instrumentation has also improved and grown as a necessary complement to space-based observations. Credits (background image): picture of the $3.6 \mathrm{~m}$ telescope at La Silla (ESO), Chile, taken by the author.

ing precise photometric measurements and parallaxes of millions of stars, the Optical Gravitational Lensing Experiment (OGLE) survey ([11, 12]), the Whole Earth Telescope (WET) survey ([13]), the Spitzer space survey ([14]), the Hubble space telescope ([15]), or the future Large Synoptic Survey Telescope (LSST, [16]), to name a few.

Today, those surveys and their legacy data are essential for the advance in the understanding of Cepheid stars (e.g., the VEGA/CHARA survey, [17]), the asteroseismology of white dwarfs ([18]), the study of Galactic archaeology ([19]), or the study of the stellar interiors of red giants with the combined data of the Apache Point Observatory Galactic Evolution Experiment (APOGEE) survey and the Kepler mission ([20]).

Today most of the data generated by those missions, instruments and networks are available by interconnected archives throughout the VO technology and standardisation protocols (e.g. CDS, Vizier, Simbad, Aladin, TOPCAT ${ }^{3}$, to name a few). These well-known general purpose VO tools have been used for decades, with no specific run modes or modules for asteroseismology. Today this is changing. Here I will give a brief description of the next generation VO tools focused on asteroseismology: the Seismic Plus and TOUCAN portals.

\section{Next generation tools for stellar pulsations}

Next generation is a terminology often used to indicate a leap forward in the technology involved in astronomical instrumentation or even in software application. Here we are dealing with a hybrid

\footnotetext{
${ }^{3}$ http://cdsweb.u-strasbg.fr; http://vizier.u-strasbg.fr/viz-bin/VizieR; http://simbad. u-strasbg.fr/simbad/; http://aladin.u-strasbg.fr/aladin.gml; http://www.star.bris.ac.uk/ mbt/ topcat/
} 
of those worlds: the technological development of the instrumentation being employed in asteroseismic studies (see previous section), and the software development under the VO to handle the data generated by that instrumentation.

In 2006 several scientific teams (24 partners) organized a network under the project HELAS (European HELio- and ASteroseismology network), as a network funded by the European Commission as part of the Sixth Framework Programme (FP6). The activities of HELAS structured this European Research Area by bringing together the European groups active in helio- and asteroseismology. Years later, once the HELAS project was finished, the partners got funded again for the Exploitation of Space Data for Innovative Helio- and Asteroseismology (SpaceInn) project ${ }^{4}$ with the objective to build on the existing European strength in the field of time-domain stellar physics. Indeed, one of the main tasks of that new project was to build a next generation VO tool able to centralize all the observational data related with stellar variability, helio and asteroseismology. This tool is the Seismic Plus portal ${ }^{5}$.

Likewise, during the SpaceInn project, ways were explored in which the observational data could efficiently communicate with theoretical models in the era of the Virtual Observatory. The solution was found in the state-of-the art development of theoretical models in the VO for asteroseismology, the TOUCAN ${ }^{6}$ portal ([21]).

\section{Seismic Plus}

The asteroseismic data has become the state-of-the-art in the research of stellar interiors, and thereby providing the most updated and unprecedentedly accurate information not only for stellar structure and evolution, but also for planetary research, which requires that the stars that host planets are accurately characterized. The Seismic Plus portal is the place that gathers an extended list of existing data sources, namely solar and stellar seismic data obtained with space missions (e.g., light curves from CoRoT Kepler, etc.) and ground-based facilities (e.g., follow-up observations of those space missions with astrometry, spectroscopy, etc.). Likewise, Seismic Plus provides online tools to exploit the data (e.g., combining the data to produce new outputs). The current implemented data sources ${ }^{7}$ can be grouped in four main types: (1) Time series. This is the main data type in asteroseismology. It contains not only information of the stellar pulsations but also may show up insights on the stellar activity or even transiting planets; (2) Spectroscopy. Stellar modeling on which asteroseismology relies requires constraints that only spectroscopic analysis (e.g., for $[\mathrm{Fe} / \mathrm{H}]$ and $T_{\text {eff }}$ estimates) can provide with sufficient accuracy to be useful in the field; (3) Stellar parameters. Observational seismic indices, based on large amount of data provided by the space missions like CoRoT and Kepler, help to characterize large numbers of stars to first order. Together with the classical photometric color indices or parallaxes, those new indices have emerged which characterize the stellar pulsation and thus the stellar structure.

The Seismic Plus portal was conceived to remain as simple as possible in all its features, from the query to the presentation of the data, without compromising its main utility, i.e. providing the most relevant information from all the linked sources. To do so, the portal has three main functions: (1) to list and describe the data sources; (2) to cross search for existing data (and data types), and

\footnotetext{
${ }^{4}$ This project with reference FP7-312844 was ended on December 2016. The HELAS consortium is now applying for European EINFRA project under $\mathrm{H} 2020$ program to improve and update the infrastructures related to the asteroseismic observations and data access.

${ }^{5}$ http://voparis-spaceinn.obspm.fr/seismic-plus/

${ }^{6}$ http://www.laeff.cab.inta-csic.es/projects/toucan3/toucan/

${ }^{7}$ Data sources and data products are in continuous evolution so this list remains open to changes in the observational landscape and in current scientific issues.
} 

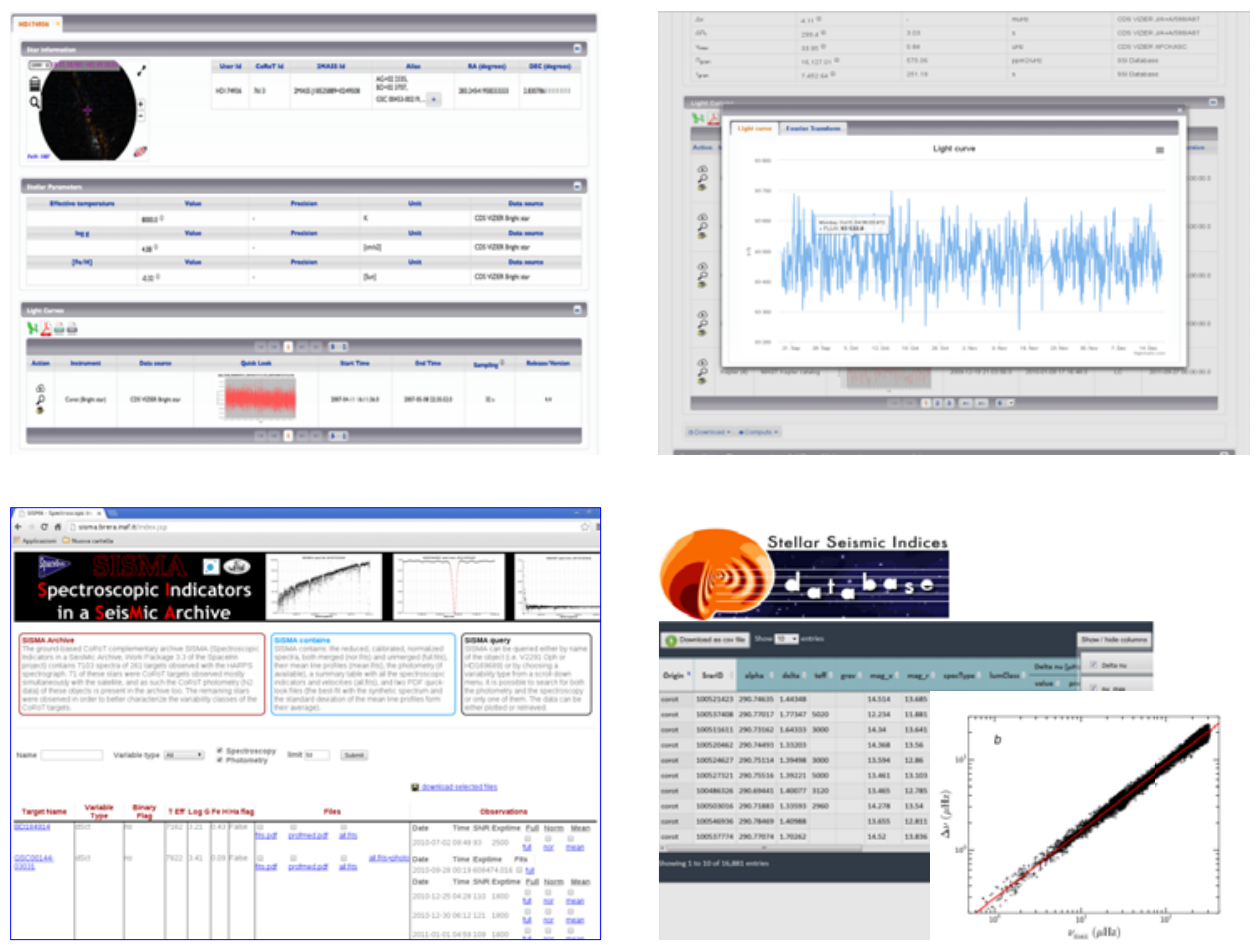

Figure 3. Screenshots of the Seismic Plus' main features: (top, left) first output for a single-star query; (top, right) high-level product consisting in a fast Fourier transform of the selected light curve; (bottom, left) main page of the SISMA (Spectroscopic Indicators in a SeisMic Archive) module. This feature calculates indicators from spectra and time series of spectra useful for asteroseismic studies; (bottom, right) main page of the SSI (Stellar Seismic Indices) module. The SSI provides asteroseismic indices commonly used in asteroseismology, like large and small separations ( $\delta v$ and $\Delta v$, repectively), the frequency of the mode with observed maximum amplitude $\left(v_{\max }\right)$, etc.

(3) to help in the more complex data queries (cross queries throughout the archives) and in the second level output generated by the tool. Two main tools are now providing second-level outputs within the portal: the SISMA ([22]) and the SSI tools ([23]) (Fig. 3).

\section{Toucan, the VO gateway to stellar models}

Nowadays, model databases are extensively used, leading to interpret theoretically large sets of observations, like those provided by the space missions and surveys (e.g., [19, 20, 24, 25]).

Such analyses require different codes, numerical approaches, generally imply different definitions of physical quantities and/or modelling parameters, different output formats, etc. The lack of homogeneity makes it difficult to design automatic tools to simultaneously work with different models and/or applications able to use the models on the fly. The VO (see Sect. 1) is the ideal frame to overcome such difficulties. TOUCAN is the first tool in the VO focused on theoretical asteroseismic models. The portal, which has been developed within the Spanish Virtual Observatory project ${ }^{8}$, pro-

\footnotetext{
${ }^{8}$ http://svo.cab.inta-csic.es, supported from the Spanish MINECO through grant AYA2014-55216.
} 

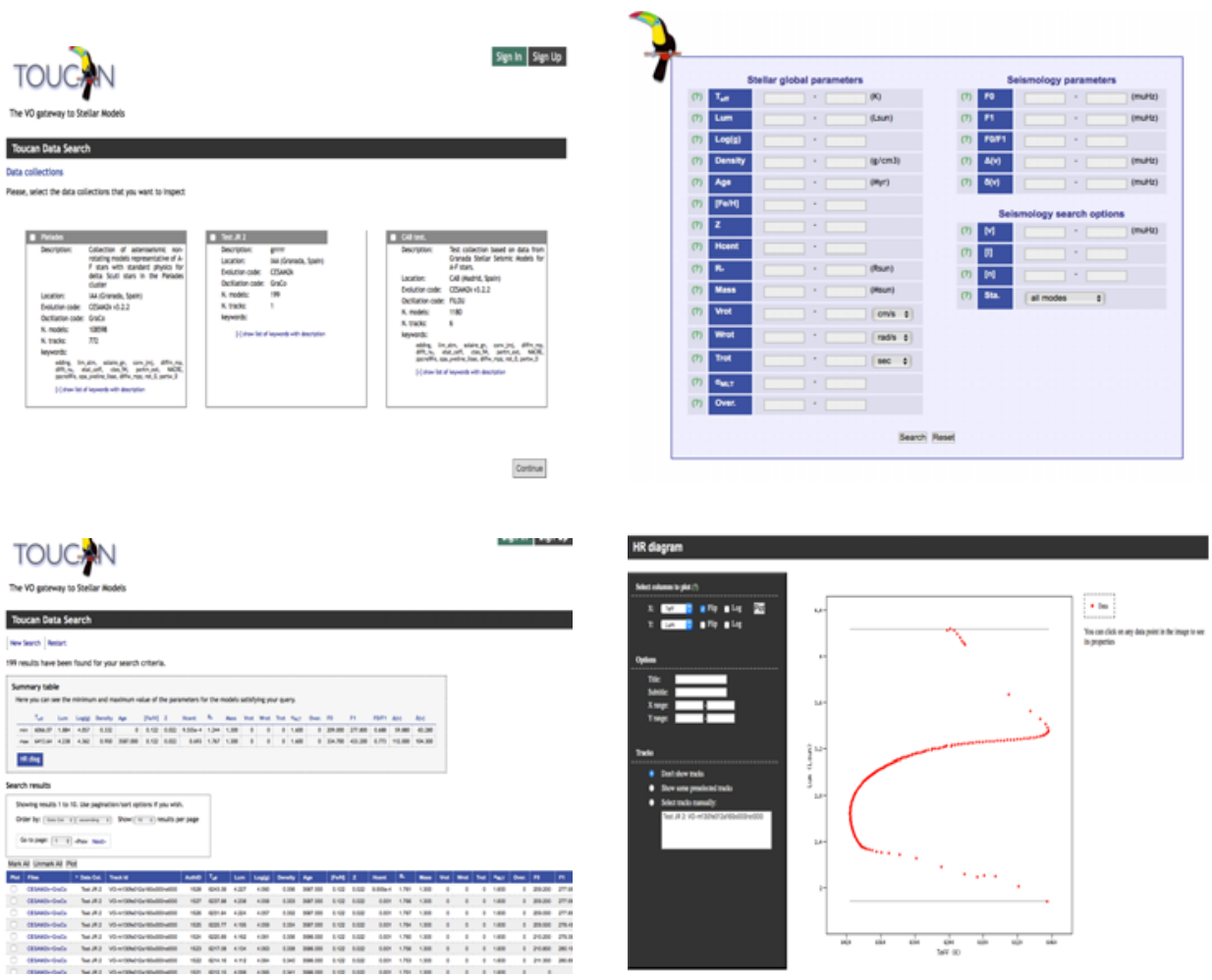

Figure 4. Screenshots of the TOUCAN's main features: (top, left) description of the Data Collections; (top, right) query of models with criteria on both structure/modelling parameters and seismic parameters; (bottom, left) example of the results provided by the TOUCAN portal: a summary table indicates the minimum and maximum values of a set of structure and seismic parameters. Below, a complete list of individual models is given; (bottom, right) plots of models selected for comparison. Online plots of both global variables (as illustrated in the panel, with an HR diagram) and shell variables (e.g., the variation of the pressure with the radius) are possible.

vides access to a database of approximately $1.5 \times 10^{6}$ models. Users can search for those models fulfilling physical criteria (structure and seismic properties), visualize the data, get some high-level products, and download models in both original format and VO tables (Fig. 4).

Currently the portal is in beta version ${ }^{9}$, running on a local prototype (i.e. asteroseismic models are stored at the portal's site). This prototype has already been used for several scientific works (see, e.g., [21, 26-30]). However, the portal is in continuous development (Fig. 5) in order to adapt the tool to a wider international context and provide access to millions of models distributed in very different and heterogeneous data collections ${ }^{10}$.

Likewise, the scientific and technical teams of Seismic Plus and TOUCAN are performing a feasibility study of the interconnection between the two portals, so that researchers and general users have access to both observations and models in an efficient and smart way, with the objective of maximizing the scientific time in effective research.

\footnotetext{
${ }^{9}$ http://svo.cab.inta-csic.es/theory/sisms3/

${ }^{10}$ There is a prototype of a new version of the tool at http://svo.cab.inta-csic.es/main/index.php.
} 


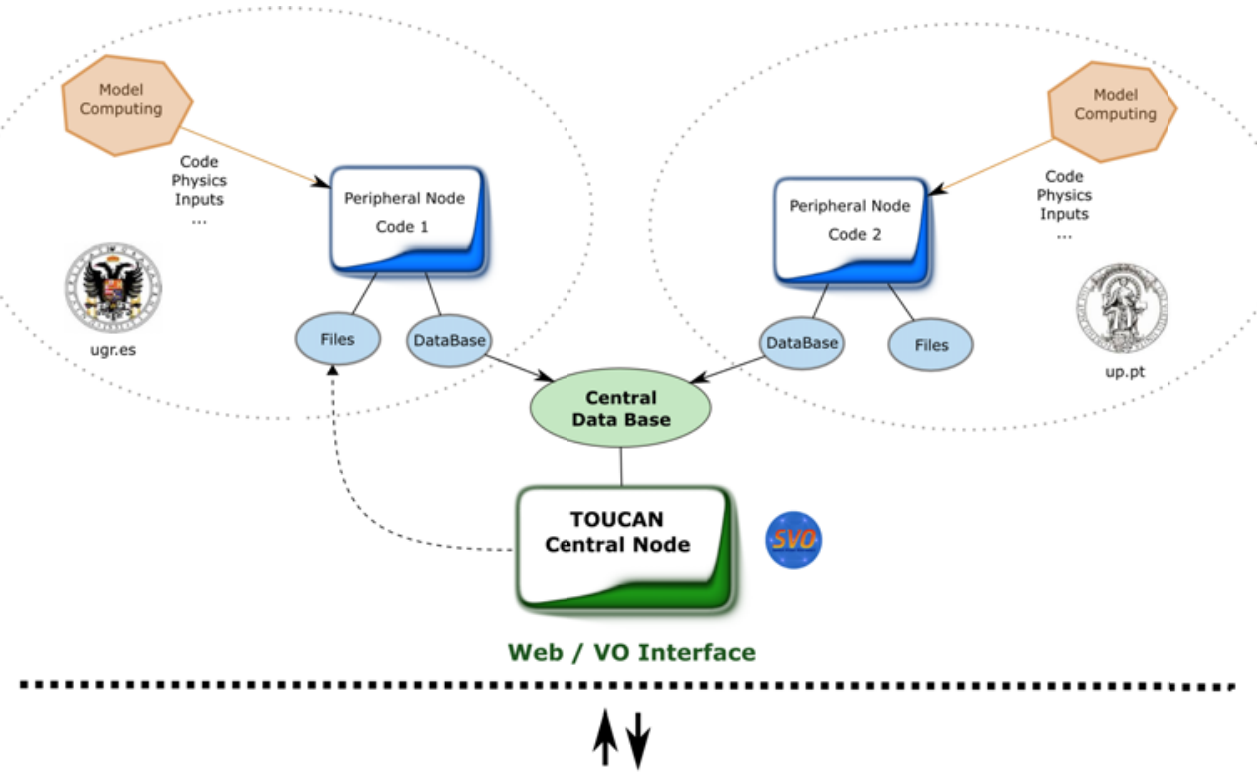

Users / Applications

Figure 5. Scheme of the new TOUCAN's client-server web architecture. Current development intends to upgrade the tool to a distributed framework. A TOUCAN central service (server) will communicate with TOUCAN peripheral services (clients). The peripheral services will contain the physically stored Data Collections. The TOUCAN central service will connect to the different peripheral services (or TOUCAN nodes) to get the information queried by the user, and will present it to the users.

Acknowledgments: I acknowledge funding support from Spanish public funds for research under project ESP2015-65712-C5-5-R (MINECO/FEDER), and under the Ramón y Cajal research fellowship with reference RYC2012-09913 (MINECO/FEDER).

\section{References}

[1] F. Genova, M.G. Allen, C. Arvise, A. Lawrence, F. Pasian, E. Solano, J. Wambsganss, Astronomy and Computing 11, 181 (2015), 1506.06567

[2] J.M. Matthews, Communications in Astroseismology 150, 333 (2007)

[3] A. Baglin, E. Michel, M. Auvergne, The seismology programme of the CoRoT space mission, in European Space Agency, (Special Publication) ESA SP (2006), Vol. 624 SP, ISBN 9290929359, ISSN 03796566, 0811.1080

[4] R.L. Gilliland, T.M. Brown, J. Christensen-Dalsgaard, H. Kjeldsen, C. Aerts, T. Appourchaux, S. Basu, T.R. Bedding, W.J. Chaplin, M.S. Cunha et al., Publications of the Astronomical Society of the Pacific 122, 131 (2010)

[5] C. Broeg, A. Fortier, D. Ehrenreich, Y. Alibert, W. Baumjohann, W. Benz, M. Deleuil, M. Gillon, A. Ivanov, R. Liseau et al., EPJ Web of Conferences 47, 03005 (2013), 1305.2270 
[6] H. Kjeldsen, TESS and the Future of Asteroseismology from Space, in These Proceedings (2017)

[7] H. Rauer, C. Catala, C. Aerts, T. Appourchaux, W. Benz, A. Brandeker, J. ChristensenDalsgaard, M. Deleuil, L. Gizon, M.J. Goupil et al., Experimental Astronomy 38, 249 (2014)

[8] M. Graham, Challenges in the Automated Classification of Variable Stars in Large Databases, in These Proceedings (2017)

[9] L. Eyer, Pulsating Star Research and the Gaia Revolution, in These Proceedings (2017)

[10] T. Metcalfe, The Impact of Gaia Parallaxes on Asteroseismic Inferences from Kepler, in These Proceedings (2017)

[11] A. Udalski, OGLE Cepheids in the Milky Way, in These Proceedings (2017)

[12] H. Netzel, Blazhki Modulation in First-Overtone RR Lyrae Stars from the OGLE Collection, in These Proceedings (2017)

[13] J. Provencal, A Survey of Pulsating DA and DB White Dwarfs with the Whole Earth Telescope, in These Proceedings (2017)

[14] P. Whitelock., Spitzer Observations of Large Amplitude Variables in IC1613, in These Proceedings (2017)

[15] K. Sokolovsky, The Hubble Catalogue of Variables, in These Proceedings (2017)

[16] M. Graham., LSST and Its Potential for Pulsating Star Research, in These Proceedings (2017)

[17] N. Nardetto, VEGA/CHARA Interferometric Observations of Cepheids, in These Proceedings (2017)

[18] S. Kepler, Pulsating White Dwarfs in Large Surveys, in These Proceedings (2017)

[19] V. Silva-Aguirre, Asteroseismology and Galactic Archaeology in the Era of Large Surveys, in These Proceedings (2017)

[20] M. Pinsonneault, APOKASC 2.0: Asteroseismology and Spectroscopy of Red Giants, in These Proceedings (2017)

[21] J.C. Suárez, A. García Hernández, A. Moya, C. Rodrigo, E. Solano, R. Garrido, J.R. Rodón, Astronomy \& Astrophysics 563, A7 (2014), 1402.0723

[22] M. Rainer, E. Poretti, A. Mistò, M.R. Panzera, M. Molinaro, F. Cepparo, M. Roth, E. Michel, M.J.P.F.G. Monteiro, AJ 152, 207 (2016), 1611.02715

[23] R. Samadi, R. De Assis Peralta, E. Michel, K. Belkacem, C. Renie, M. Abed, EPJW, in press (2016)

[24] P. De Cat, Pulsating Variable Stars and Large Spectroscopic Surveys, in These Proceedings (2017)

[25] I. Soszyński, The Impact of Large-Scle, Long-Term OPtical Surveys on Pulsating Star Research, in These Proceedings (2017)

[26] A. García Hernández, A. Moya, E. Michel, R. Garrido, J.C. Suárez, E. Rodríguez, P.J. Amado, S. Martín-Ruiz, A. Rolland, E. Poretti et al., Astronomy and Astrophysics 506, 79 (2009)

[27] E. Poretti, E. Michel, R. Garrido, L. Lefèvre, L. Mantegazza, M. Rainer, E. Rodríguez, K. Uytterhoeven, P.J. Amado, S. Martín-Ruiz et al., Astronomy and Astrophysics 506, 85 (2009)

[28] L. Mantegazza, E. Poretti, E. Michel, M. Rainer, F. Baudin, A. García Hernández, T. Semaan, M. Alvarez, P.J. Amado, R. Garrido et al., Astronomy \& Astrophysics 542, A24 (2012)

[29] A. García Hernández, A. Moya, E. Michel, J.C. Suárez, E. Poretti, S. Martín-Ruíz, P.J. Amado, R. Garrido, E. Rodríguez, M. Rainer et al., Astronomy \& Astrophysics 559, A63 (2013)

[30] A. García Hernández, S. Martín-Ruiz, M.J.P.F.G. Monteiro, J.C. Suárez, D.R. Reese, J. PascualGranado, R. Garrido, ApJL 811, L29 (2015), 1509.01111 